\title{
The Influence of Diet upon the Teeth.
}

$\mathrm{O}$ WING to the prevalence of dental caries under our present conditions of living and dieting, knowledge of its cause, and the means by which it may be prevented or its ravages mitigated, is of great importance not only to the individual but also to the nation. During the last decade, attention has been directed by a number of observers to the influence which various types of diet may exert upon the structure of the teeth, often in conjunction with their effects upon other tissues of the body, especially the skeleton. This work has demonstrated that diet plays an important part in producing abnormalities of the teeth of experimental animals: the relationship of these ab. normalities to dental caries is not so clear, since animals comparatively rarely suffer from this type of dental disease. Some evidence has been produced that there is a definite relationship between the structure of the teeth and caries, but it has not met with universal acceptance. In this article the influence of diet upon the structure of the teeth will be more especially considered, and the problem of dental caries only mentioned incidentally, where it appears to throw light upon the manner in which diet may affect the teeth. To illustrate the points raised, reference will be made to a few of the more recent papers dealing with the subject: further details will be found in these papers and also in those mentioned in their bibliographies.

There appear to be three ways in which diet can affect the teeth : food lodged in their crevices may undergo changes which lead to alterations in the enamel and other structures of the tooth; the diet may alter the composition of the saliva and thereby affect the teeth indirectly; or it may alter the structure of the tooth directly, in a comparable manner to its effect upon other tissues of the body. In the first two cases the agent acting upon the teeth attacks them, so to speak, from their outer or superficial surface; in the last, the changes depend upon an alteration in the composition of the blood and lymph reaching the tooth, and are thus initiated from the pulp or from within the tooth itself.

There is probably general agreement that caries is initiated by the fermentation of carbohydrate food stagnating between the cusps of the molar teeth, between the teeth themselves, and round their neeks. According to J. Sim Wallace (Med. Press and Circ., vol. 124, p. 487 ; 1927), diet cleanses the teeth mechanically, by initiating the secretion of ptyalin and by controlling the bacterial flora which digests remnants of food remaining between them. Efficient mastication is necessary both for keeping them clean and for ensuring their proper development together with that of the jaws. Inefficient mastication, leaving stagnating mucus on and around the teeth, encourages the development of tartar, since bacterial decomposition of the mucus leads to the production of alkali and the consequent precipitation of certain salts. On the other hand, the consumption of soft, sticky carbohydrate food leads to the production of acid, from the bacterial decomposition of the masses adhering to the crevices of the teeth, and this acid attacks the interprismatic areas of the enamel, initiating caries. Thus starchy or sugary foods favour the development of caries, whilst the more fibrous foods, such as fruits or raw vegetables, and fish and meats, tend to cleanse the teeth both mechanically and by increasing the secretion of saliva which washes away adhering remnants, and thus hinder or prevent dental decay. Experiments have shown that acidforming bacteria can initiate caries when grown in vitro in the presence of teeth, the acid attacking the enamel: at least two organisms have been found in or on carious teeth which can form acid from carbohydrate, but whether either of them is specific for this disease is not yet certain. Penetration of the enamel is very slow unless the acidity is of the order of $p \mathrm{H} \mathbf{5 . 0}$ (I. H. Maclean, Proc. Roy. Soc. Med., vol. 20 (Sect. Odont.), p. 873 ; 1927).

Little is known about the possibility of altering the composition of the saliva by changes in the diet. Although saliva is a secretion, it is possible that certain changes in the composition of the blood may be reflected in this secretion : in health, the variations in the different constituents of the blood lie within very narrow limits, but they are more marked in disease, and variations from the average normal might then be observed in the composition of the saliva. In fact, C. L. Pattison has found definite changes in the calcium with variations in the diet (Brit. Med. Jour., vol. 2, p. 6; 1926). The work was carried out on children suffering from tuberculosis of the bones and joints. On admission to hospital the average salivary calcium was $4.77 \mathrm{mgm}$. per 100 c.c. On the ordinary hospital diet this rose to $7.79 \mathrm{mgm}$. ; on a diet containing little milk, no cod-liver oil or eggs, but oatmeal and olive oil, the figure was almost unchanged ( $4.68 \mathrm{mgm}$.); but when plenty of milk and cod-liver oil and an egg, but no oatmeal, were given daily, the salivary calcium rose to $10.68 \mathrm{mgm}$. per 100 c.c. In the light of our present knowledge, there is little doubt that these results are to be explained by the improvement in absorp. tion and retention of calcium which occurs on the administration of adequate amounts of vitamin $\mathbf{D}$, following a relative or absolute deficiency in the intake of this compound. Addition of a calcium salt to the diet for a short period was without effect on the salivary calcium. Whether changes in the composition of the saliva can influence the teeth is not known : a possible indirect effect might be suggested by means of subsequent alterations in the bacterial flora of the mouth.

It is now known that the structure and composition of the teeth can be affected by changes in the amounts of calcium and phosphorus and of vitamins $\mathrm{C}$ or $\mathrm{D}$ in the diet : in general, it may be said that the proper development of the teeth usually shows a close relationship to the proper development of the bony skeleton. In producing imperfect calcification and structure of the teeth, deficient intake of calcium or phosphorus, or an improper ratio between these two elements in the diet, has most often been associated with a deficiency of vitamin $D$.

No. 3044, VoL. 121] 
also. J. A. Marshall, however, found that pups exposed to sunlight and kept on a diet containing an adequate amount of vitamins, but with improper quantities of calcium and phosphorus, developed gross changes in their teeth, including imperfect calcification of the enamel and relative absence of the dentine: there was marked delay in dentition, and the deciduous teeth were not shed (Jour. Amer. Med. Ass., vol. 81, p. 1665; 1923). G. Toverud also observed that in white rats maintained on a diet deficient in calcium the teeth contained less mineral ash than normal teeth (Jour. Biol. Chem., vol. 58, p. 583; 1923) ; whilst M. R. Jones and F. V. Simonton found that the addition of alkali (sodium carbonate) to a diet complete in every other respect produced changes in the teeth and jaws of puppies (Proc. Soc. Exper. Biol. and Med., vol. 23, p. 734 ; 1926). It is known that the absorption of calcium and phosphorus is influenced by the hydrogen ion concentration of the contents of the intestine, alkali interfering with this absorption and acid increasing it. C. J. Grieves (Jour. Amer. Med. Ass., vol. 79, p. $1567 ; 1922$ ) also found that a deficiency of calcium in the diet increased the amount of dental caries in the teeth of rats. Decay also occurred when the calcium was increased above the optimal amount: thus the effective factor appeared to be an improper calcium-phosphorus ratio rather than a simple change in the amount of calcium alone.

Among the symptoms of scurvy, due to deficiency of vitamin $\mathrm{C}$ in the diet, are hæmorrhages in various parts of the body, including the gums, in which situation they are accompanied by a loosening of the teeth in the jaws. Experimentally, changes in the teeth have been described by several authors: P. R. Howe (Jour. Amer. Med. Ass., vol. 79, p. $1565 ; 1922)$, has observed decalcification of guineapigs' teeth, when the animals were kept on a scorbutic diet, with recalcification when a source of vitamin $\mathrm{C}$, such as orange juice, was added to the food. Dental caries was present in the animals on the faulty diet. F. M. Wells (Proc. Roy. Soc. Med., vol. 12 (Sect. Odont.), p. 22; 1919) and Toverud (loc. cit.) have described changes in the pulp of the teeth of scorbutic guinea-pigs with replacement of the orthodentine by osteodentine: the degeneration of the pulp may occur before other clinical signs of scurvy are present.

Probably the most important factor in producing alterations in the teeth is a shortage of vitamin $\mathrm{D}$, or the antirachitic vitamin, with or without accompanying defects in the diet, such as variations in the calcium-phosphorus ratio. E. V. McCollum and his collaborators described changes in the teeth of rats maintained on diets low in protein, calcium, and fat-soluble vitamins (Bull. Johns Hopkins Hosp., vol. 33, p. 202 ; 1922), and Grieves (loc. cit.) found that caries was worse in rats when the diet was deficient in fat-soluble vitamin as well as calcium than when it was deficient in calcium alone. In puppies, May Mellanby has shown that the most important variables affecting the formation of the teeth are variations in the amount and type of cereal in the food, in the amount of fat-soluble vitamin present, and in the degree of exposure of the animals to ultra-violet light (M. Mellanby, Proc. Roy. Soc. Med., vol. 16 (Sect. Odont.), p. 74; 1923, etc., summarised by E. Mellanby, Brit. Med. Jour., vol. 1, p. 515; 1926). The less vitamin and the more cereal present in the diet, the worse was the structure of the teeth. When the bitch was fed on the deficient diet during pregnancy and lactation, the deciduous teeth of the pups were affected; if the latter were given the diet after weaning, the defects were disclosed in the permanent teeth. It thus seems possible that the influence of carbohydrates upon dental caries is not only due to their fermentation in the mouth, but also to their effect, in association with the fat-soluble vitamin, and after their digestion and absorption, upon the structure of the teeth.

How far are these results applicable to human beings? It is beyond the scope of this article to discuss the relationship between the structure of human teeth and dental caries, but it may be mentioned that May Mellanby has adduced evidence of a close relationship between defective structure and caries, based on the microscopic examination of a large number of teeth. Discrepancies are explained as due to alterations in the structure of the teeth after they have erupted. That the defective structure is due to deficiencies in the diet is possible but unproven, since the diets of the patients from whom the teeth were obtained were not known or regulated ; but where it has been possible to control the diet, a definite relationship between it and the spread of caries has been observed. About thirty children in an institution were divided into three groups, and each group given a carefully selected diet (May Mellanby, C. L. Pattison, and J. M. Proud, Brit. Med. Jour., vol. 2, p. 354; 1924). Careful examination of the teeth was made at the beginning and end of the experimental period of seven to eight months, and the general condition as regards defective structure and the position and extent of caries noted. One of the diets was the ordinary hospital diet : on this the average number of teeth per child in which caries had spread was 2.9 ; on a diet containing less milk and no butter, but more oatmeal, i.e. low in calcifying vitamin (vitamin D) and calcium, the spread of caries was represented by $\mathbf{5 \cdot 1}$ teeth becoming more affected in each child; whilst on a diet containing more milk and no oatmeal, i.e. abundant vitamin $\mathrm{D}$ and calcium, the figure was 1.4 only. Thus there appears to be a close relationship between the diet and the spread of dental caries in human beings.

More recently, May Mellanby has published some work on the structure of human teeth, the results of which suggest that diet plays an important part in producing defects of structure (Brit. Dental Jour., July 1, 1927). More than 1000 deciduous and more than 250 permanent teeth were examined both macroscopically and microscopically: in many cases the first examination was made whilst they were still in the mouth. The results obtained depend, of course, upon the definitions of the terms 'normal' and 'abnormal' as regards tooth structure. Descriptions are given of the naked-eye

No. 3044, VoL. 121] 
appearance of the enamel in various degrees of hypoplasia, and of the microscopic picture of the dentine : any uncalcified areas in the dentine are considered to be abnormal, but are so common that it is possible some authorities would consider them normal. Close agreement between the macroscopic and microscopic appearances was obtained in more than 85 per cent. of the examinations. Of the deciduous teeth, 14 per cent. were found to be perfectly calcified, 21 per cent. were slightly, and 64 per cent. definitely, hypoplastic. The incisors were the best calcified, 49 per cent. being normal : 8 per cent. of the canines, 7 per cent. of the first molars, and only 1 per cent. of the second molars could be so classed. Slighter defects appeared among the remaining incisors and canines, and the more severe degrees of hypoplasia were observed in the majority of the defective molars. The teeth obtained from private sources, as opposed to those from dental clinics, were less defective in structure. Of the permanent teeth examined, none was normal, and 92 per cent. showed definite hypoplasia; but these figures give no indication of the condition of permanent teeth in Great Britain, since all those examined had been extracted for caries, or to help in the adjustment of irregularities.

These results also indicate that there is less chance of interference with calcification before birth than afterwards, and that the more rapid the development of the tooth, the more defective is its structure likely to be. The defects in structure are probably to be correlated with the diet during the time the teeth are developing in the jaws: thus before birth, even if the mother is on a deficient diet, the foetus can obtain its requirements of salts and vitamins by the sacrifice of the maternal stores. After weaning, the child has to depend entirely on its food and on its own stores, which are likely to be low if the mother's diet has been poor, for its supply of the substances necessary for the proper development and calcification of the teeth. At the same time, the diet frequently contains a large amount of cereal products, the influence of which is exerted against proper calcification. The incisors are the most advanced in development at birth, and are also the best developed structurally. The second molars are the least developed at birth; they grow rapidly after birth, and have the worst structure of any of the teeth.

In conclusion, it may be said that diet affects the teeth as it affects the other tissues of the body, and that the teeth, like the other tissues, respond more easily to some defects in the diet than to others, but that the same defect rarely affects more than a few of the tissues to a marked degree. In addition, the diet can affect the teeth locally by causing alterations in their environment, the changes in the teeth then starting from their oral surfaces.

The degree to which diet directly affects the structure of the teeth depends in part on the definition of the terms 'normal' and 'abnormal' as regards this structure : and on this depends again the relationship between abnormality of structure and dental decay. In any case, caries will not be initiated unless acid is present on the oral surfaces of the tooth. It may be pointed out that it is difficult to produce caries in animals unless at the same time the conditions are such as to lead to defective structure also.

\section{The Enhancement Principle in X-ray Photographs.}

\section{By Sir William BragG, K.B.E., F.R.S.}

$\mathrm{A}^{\mathrm{N}}$ interesting phenomenon is often shown in the X-ray rotation photographs of crystals. Photographs of this kind are obtained by causing a crystal to revolve steadily about an axis perpendicular to the direction of a fine pencil of homogeneous X-rays. As the crystal revolves, one set of planes after another comes to a favourable condition for reflecting the pencil and a corresponding spot appears upon the plate. The crystal is usually very small indeed, and the shape of the crystal largely determines the form of the spot. The complete photograph shows an array of spots which displays certain regularities of arrangement as exemplified in Figs. 1, 2, and 3. When the photographic plate is flat, the spots arrange themselves on hyperbolæ as in Fig. 1 ; if a circular film is used the hyperbolæ are replaced by straight lines as in Figs. 2 and 3. The phenomenon to which attention is now directed consists in the enhancement, sometimes a very great enhancement, of certain groups of the spots. The explanation is more readily understood if consideration is first given to an analogous case of greater simplicity.

An ordinary optical grating gives a series of spectra : if the incident light is homogeneous, each spectrum is limited to a line. If now every fifth line in the grating were intensified or altered in some way, there would be added to the series already referred to a second series consisting of lines five times as close-packed, and every fifth line of the new spectrum would coincide with one of the old. For the sake of what follows, this may be put in another way.

If, in the first place, the grating had contained only lines corresponding to those which we have spoken of above as being intensified in some way, the more numerous series of spectra also referred to above would have appeared upon the plate. If, now, four other lines were intercalated uniformly between the lines already drawn upon the grating, every fifth line of the series of spectra would be enhanced.

From this simple case we may now proceed to the more complicated three-dimensional case of the crystal. A first example may be taken from the work on the structure of the silicates which has been carried out by Prof. W. L. Bragg and his colleagues at Manchester. They have shown that a silicate may be regarded in the first instance as a compilation of close-packed spherical oxygen atoms : the other atoms belonging to the silicate are to be thought of as inserted in the interstices in the

No. 3044, VoL. 121] 